\title{
Label-free and spectral-analysis-free detection of neuropsychiatric disease biomarkers using an ion- sensitive GaInAsP nanolaser biosensor
}

\author{
Keisuke Watanabe ${ }^{\mathrm{a},}$,, Munetaka Nomoto ${ }^{\mathrm{b}}$, Fumio Nakamura ${ }^{\mathrm{b}}$, Shoji Hachuda ${ }^{\mathrm{a}}$, Akihiro Sakata ${ }^{\mathrm{a}}$, Takumi \\ Watanabe $^{\mathrm{a}}$, Yoshio Goshima ${ }^{\mathrm{b}}$, and Toshihiko Baba ${ }^{\mathrm{a}}$ \\ aDepartment of Electrical and Computer Engineering, Yokohama National University \\ ${ }^{b}$ Department of Molecular Pharmacology and Neurobiology, Yokohama City University \\ ${ }^{*}$ Corresponding author: Keisuke Watanabe \\ Address: 79-5 Tokiwadai, Hodogaya, Yokohama 240-8501, Japan \\ Email address: watanabe-keisuke-zx@ynu.jp \\ Telephone number: +81-45-339-4257
}

\begin{abstract}
The emission intensity of GaInAsP semiconductors that show an ion sensitivity is altered by the surface charge. In this study, we propose a biosensing technique using GaInAsP photonic crystal nanolasers based on this principle. Here, simple and rapid detection of collapsin response mediator protein 2 (CRMP2) is demonstrated, which is a promising biomarker candidate for neuropsychiatric diseases existing in peripheral white blood cells. We prepared CRMP2 as a standard protein and introduced sodium dodecyl sulfate (SDS) as an anionic surfactant to enhance the net negative charge of the protein. The nanolaser was modified in advance with an anti-CRMP2 antibody and then photopumped at a constant power. The laser emission intensity was monitored during the antibody-antigen reaction. Consequently, CRMP2 was detected as a decrease in the emission intensity. We achieved a limit of detection of $3.8 \mu \mathrm{g} / \mathrm{mL}$ that satisfies the requirement for clinical biomarker testing. Without the requirements of any kind of labels and spectral analyses, this technique allows for simple, rapid, and low-cost biomarker detection.
\end{abstract}

Keywords: GaInAsP; label-free; photonic crystal; nanolaser; biomarker; collapsin response mediator protein

\section{Introduction}

Biomarker tests as quantitative clinical indicators are useful for the early detection and evaluation of progress and treatment effects of severe diseases. Neuropsychiatric diseases, including schizophrenia, are mainly diagnosed through clinical interviews based on the Diagnostic and Statistical Manual of Mental Disorders, 5th Edition (DSM-5), which is de facto the world's standard diagnostic criteria (Tandon et al., 2013). However, some challenges exist because of the complicated and multiple medical conditions of patients (Weickert et al., 2013). Therefore, there are increasing demands for biomarkers and detection methods of neuropsychiatric diseases to achieve high accuracy and precision. Collapsin response mediator protein 2 (CRMP2) mediates the axon guidance signaling of Sema3A (Goshima et al., 1995) and plays an important role in the formation of neural networks and sustenance in the nervous system (Tessier-Lavigne and Goodman, 1996; Yamashita et al., 2012). The disordering of this role is thought to be deeply involved in the pathogenesis of neurodegenerative and psychiatric diseases, such as Alzheimer's disease and schizophrenia (Hong et al., 2005; Cole et al., 2007; Isono et al., 2013). Although CRMP2 is predominantly expressed in the central nervous system, and its expression in peripheral blood has also been confirmed recently, and the amount of CRMP2 varies depending on the age of patients with schizophrenia (Nomoto, 2018). We also found that several tens to several hundred femtomoles of CRMP2 is expressed in a peripheral white blood cell, which translates to a concentration of several micrograms per milliliter. If CRMP2 of this concentration scale can be easily detected, the diagnosis from the peripheral blood of a patient will become available with few burdens.

The well-known and widely used enzyme-linked immunosorbent assay (ELISA) has the advantages of high accuracy and precision. The procedure, however, is complicated and time-consuming. Immunochromatography and the lateral flow assay are much simpler than ELISA, where one can obtain the results instantly. However, they are qualitative and less accurate (Koczula and Gallotta, 2016; Sajid et al., 2015). There is a trade-off between the measurement time and the accuracy. Therefore, detection methods satisfying both requirements are desired (Hong et al., 2012; Makowski and Ivanisevic, 2011; Xavier et al., 2018). We have focused on label-free biosensors and developed a GaInAsP semiconductor photonic crystal (PC) nanolaser biosensor that can detect 
the adsorption of biomolecules from the shift of the laser wavelength (Kita et al., 2011a). For example, we demonstrated the detection of proteins such as prostate-specific antigen (PSA) with ultra-high sensitivity (Hachuda et al., 2016). In general, the wavelength shift of photonic biosensors based on optical micro/nanocavities is thought to reflect the environmental index change accompanied by protein adsorption (Chow et al., 2004; Vollmer et al., 2002), while we found that the emission intensity of the nanolaser depends on the surface charge due to the modified surface recombination, and we demonstrated the simultaneous detection of the environmental index and the surface charge (Watanabe et al., 2015). In addition, we recently observed some evidence that the wavelength shift which has been considered to arise only from the environmental index change also reflects the surface charge (T. Watanabe et al., 2017). We call such sensitivity to the surface charge ion sensitivity and such sensing iontronic sensing.

Now, we focus on the ion sensitivity in the emission intensity of the GaInAsP nanolaser rather than the wavelength and propose a simple iontronic biosensing method using neither labels nor spectral analyses (K. Watanabe et al., 2017). As iontronic biosensors based on electronics, ion-sensitive field effect transistors (ISFETs) and light addressable potentiometric sensors (LAPSs) are well-known devices that exploit the ionsensitivity in electrical signals (Makowski and Ivanisevic, 2011; Yoshinobu et al., 2005). As those based on photonics, detecting the modified photoluminescence (PL) has also been studied for GaAs (Budz et al., 2010; Nazemi et al., 2015; Tang et al., 2013), GaN (Wallys et al., 2012), CdS(e) (Ellis et al., 1997), ZnO (Viter et al., 2018), and GaInAsP (Sakemoto et al., 2016). In this method, the device preparation and measurement are much easier than the above electronic devices. Our proposed method of using the nanolaser works with a phenomenon similar to this, but its sensitivity and signal intensity are enhanced by the laser operation. In this paper, we describe this device in detail as a simple and quick biomarker tester and demonstrate the practical performance for the CRMP2 detection.
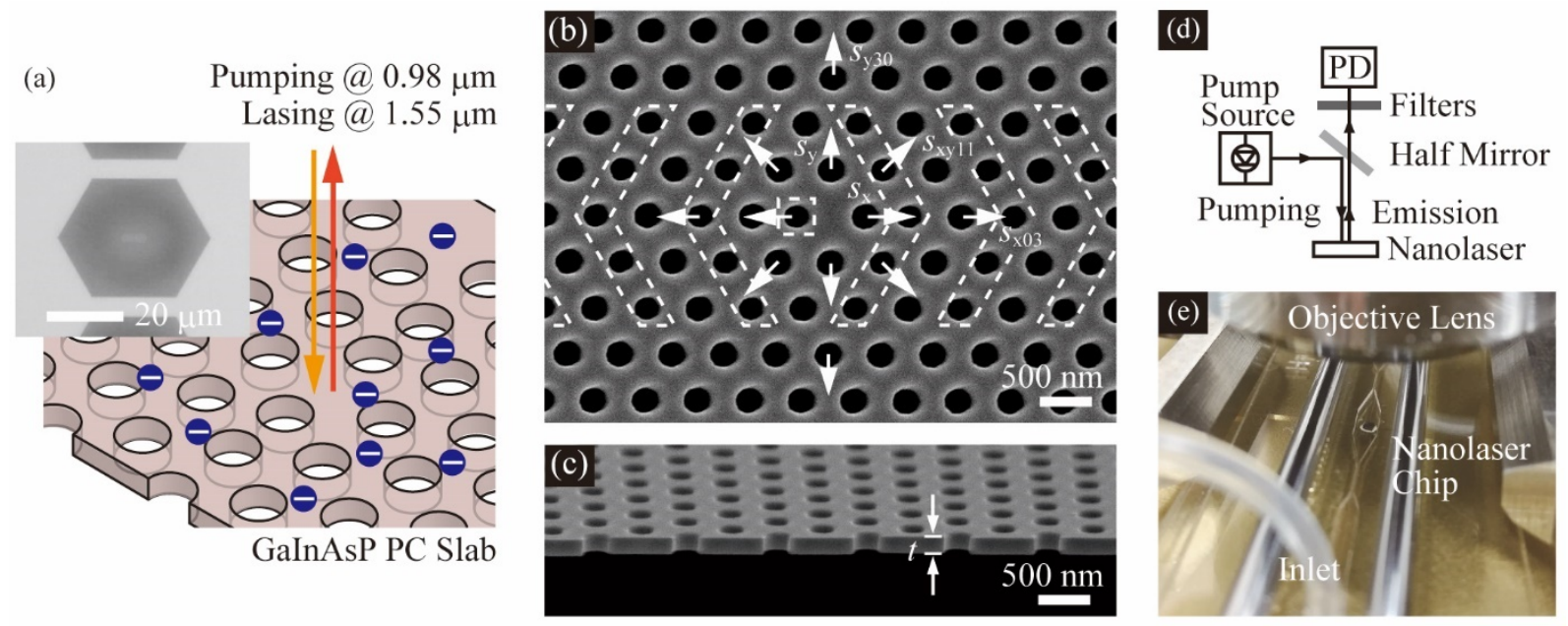

Fig. 1. GaInAsP nanolaser and measurement setup. (a) A schematic of the ion-sensitive GaInAsP nanolaser. The inset shows a top view of one nanolaser. The purple spheres show an example where a negative charge is adsorbed. (b) Top SEM view around the nanocavity. (c) Cross-sectional SEM view of the PC slab. Arrows indicate position shifted air holes and dotted lines indicate diameter modulated air holes. (d) Measurement setup. (e) The appearance of the measurement using the PDMS microfluidic channel.

\section{Materials and methods}

\subsection{Fabrication of the nanolaser and measurement setup}

The PC nanolaser is a small laser operated by photopumping (Kita et al., 2011b). As shown in Fig. 1, it consists of a GaInAsP semiconductor single-quantum-well thin membrane having weak n-type conductivity and an air-bridge-type PC structure. The periodic air holes with a diameter of $250 \mathrm{~nm}$ and a pitch of $500 \mathrm{~nm}$ were arranged in a triangular lattice. At the PC center, H0-type nanocavity, a kind of PC cavities which is formed by shifting some air holes with no missing air holes, was formed. The air-bridge membrane thickness $t$ was $\sim 180$ $\mathrm{nm}$. The total diameter of the nanolaser, including all air holes, was $\sim 40 \mu \mathrm{m}$.

The fabrication process was as follows. First, e-beam lithography (ELS-7500, Elionix) was performed to 
draw the nanolaser pattern into an e-beam resist (ZEP-520A, Zeon) on an epiwafer. Then, the pattern was transferred to the semiconductor by HI inductively coupled plasma etching (RIE-200ip, Samco). The air-bridge structure was formed by $\mathrm{HCl}$ selective wet etching. Finally, $\sim 3 \mathrm{~nm} \mathrm{ZrO}_{2}$ was deposited by atomic layer deposition (ALD) (Savannah, Ultratech/Cambridge Nanotech) to ensure the chemical stability (Watanabe et al., 2015).

The scanning electron micrographs (SEMs) of the fabricated device are shown in Figs. 1(b) and 1(c). Here, the diameter of the air holes in the region as shown by the dotted lines was made slightly smaller than others to induce an asymmetry into the cavity. Such modulation of air holes makes the radiation pattern of the laser emission single peaked and improves the coupling efficiency into the objective lens (Narimatsu et al., 2012). In this study, we further optimized the shift amount of air holes around the cavity to obtain a high quality factor $\mathrm{Q}$ while maintaining the coupling efficiency, using the three-dimensional finite-difference time-domain method, such that $s_{\mathrm{x}}=120 \mathrm{~nm}, s_{\mathrm{y}}=60 \mathrm{~nm}, s_{\mathrm{y} 11}=20 \mathrm{~nm}, s_{\mathrm{x} 03}=20 \mathrm{~nm}$, and $s_{\mathrm{y} 30}=-20 \mathrm{~nm}$, as indicated by arrows in Fig. $1(b)$.

The measurement setup is depicted in Fig. 1(d). Pulsed pump light at $0.98 \mu \mathrm{m}$ was irradiated from above via a $50 \times$ objective lens (numerical aperture of 0.55 ). The emitted light from the nanolaser was observed by a power meter (Q8383, Advantest) and/or an InGaAs near-infrared camera (Alpha NIR, Indigo Systems) via a long-pass filter that eliminated the pump light. For the pumping and measurement, we particularly used an optical fiber with a large core diameter of $200 \mu \mathrm{m}$ so that the pump spot fully covered the device area. This approach stabilized the emission intensity for small displacement of the device. To further reduce the intensity variation caused by thermal fluctuations and so on, we obtained an average intensity from 10 times acquisition. As shown in Fig. 1(e), the injection and discharge of analyte solutions were carried out by setting the nanolaser chip inside a polydimethylsiloxane (PDMS) microfluidic channel, while the emission intensity was measured. To evaluate the fundamental electrochemical properties of the emission, we prepared solutions with different $\mathrm{pH}$ values by adding $\mathrm{HCl}$ or $\mathrm{KOH}$ to a $100 \mathrm{mM} \mathrm{KCl}$ solution.

\subsection{Surface functionalization and detection method of CRMP2}

The procedure of surface functionalization of the nanolaser which was employed in this study is shown in Fig. 2. First, a self-assembled monolayer 3-aminopropyltriethoxysilane (APTES) was formed on the $\mathrm{ZrO}_{2}-$ coated nanolaser using the same ALD chamber. Then, the device was functionalized with glutaraldehyde for 1 $\mathrm{h}$ at $37^{\circ} \mathrm{C}$, followed by rinsing for $10 \mathrm{~min}$ using ultrapure water and immersing in an anti-CRMP2 monoclonal antibody solution $\left(9 \mathrm{~F}, 4 \mu \mathrm{g} / \mathrm{mL}\right.$ ) at $4^{\circ} \mathrm{C}$ overnight. Finally, the device was set in a PDMS microfluidic channel. By injecting CRMP2 solutions of different concentrations in the order from low to high, the emission intensity was measured continuously to observe the detection of CRMP2 and to evaluate its limit concentration. We also prepared a device modified with an anti-PSA antibody (4D10) as a control and also tried to detect CRMP2.

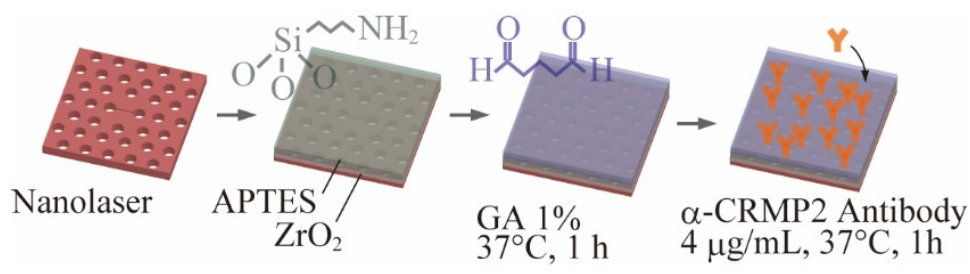

Fig. 2. Surface functionalization for CRMP2 detection.

We confirmed in another experiment that the adsorption of CRMP2 was hindered when the common phosphate buffered saline (PBS) was used as the protein solution. This result is in agreement with the reported phenomena where protein adsorption to an oxide surface is reduced in the PBS solution (Kurrat et al., 1997; Moulton et al., 2003; Wei et al., 2009). We consider that this behavior arises from a phenomenon where phosphate ions in PBS form a bidentate ligand on $\mathrm{ZrO}_{2}$ (Spielbauer et al., 1997), which is not completely covered with APTES and glutaraldehyde but exposed to the solution. Then, this ligand charges the surface negatively and gives rise to electrostatic repulsion against negatively charged CRMP2 (Moulton et al., 2003; Wei et al., 2009). To avoid this situation, we chose the 2-( $N$-morpholino)ethanesulfonic acid (MES) buffer in this experiment, which does not form such a specific ligand. Here, the charge apart from the surface beyond the Debye length is screened by counterions. We diluted the MES buffer solution (MES $100 \mathrm{mM}, \mathrm{pH}$ 6.8) to $1 \mathrm{mM}$ 
so that the Debye length is increased, and charge effect of CRMP2 enhanced after the following treatment efficiently reaches the surface and eventually modifies the emission intensity.

\subsection{Signal amplification by anionic surfactant treatment}

The nanolaser detects the change in the surface charge density after the protein adsorption. However, CRMP2 has an isoelectric point of $\sim 6$ and is almost electrically neutral in the MES buffer, resulting in a weak charge effect on the nanolaser. Therefore, we added sodium dodecyl sulfate (SDS), a kind of anionic surfactant, forcing CRMP2 to be negatively charged. Proteins which react with SDS maintain the negative charge even after the removal of the excess SDS in the solution (Hideshima et al., 2016). Therefore, we can anticipate that SDS will improve sensitivity to protein adsorption. In this process, $1 \mathrm{mg} / \mathrm{mL}$ CRMP2 solution with $0.1-1 \%$ SDS was first prepared by mixing them, followed by a heat block in a $100^{\circ} \mathrm{C}$ water bath for $5 \mathrm{~min}$. Then, excess SDS in the solution was removed by a commercially available kit (SDS-Out SDS Precipitation Kit, Thermo Scientific).

\subsection{Production of CRMP2}

Standard CRMP2 as a detection target was produced via a biochemical approach, as shown in Fig. 3(a). First, CRMP2-encoded region of the human brain cDNA library, a set of complementary DNA produced by reverse transcription of messenger RNA, was amplified using a polymerase chain reaction (PCR). Secondly, the CRMP2 plasmid was made by the ligation of a pGEX (pGEX-6p-1, GE Healthcare) vector and the PCR products using a restriction enzyme. Thirdly, the plasmid was transformed into E. coli BL21 cells followed by scaling up. Subsequently, protein expression was induced with isopropyl $\beta$-D-1-thiogalactopyranoside (IPTG). Finally, a large amount of CRMP2 expressed in the cells was harvested and purified. The purified CRMP2 was separated by SDS-polyacrylamide gel electrophoresis (PAGE), whereas the gel was stained by Coomassie Brilliant Blue (CBB), as shown in Fig. 3(b). The full length of CRMP2 is known to be $62 \mathrm{kDa}$, and the obtained band was in good agreement with this value. Thus, we confirmed that the purification process was correctly carried out. Figure 3(c) shows the result of western blotting (WB) using the anti-CRMP2 antibody (9F). We also confirmed the specificity of the CRMP2 sample. The final CRMP2 concentration for the detection experiment was determined by the Bradford protein assay.

(a)
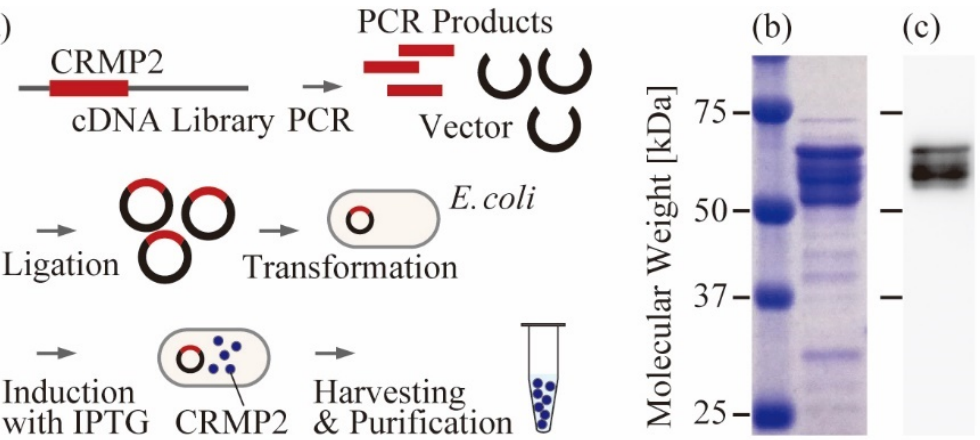

Fig. 3. The procedure of production and purification of CRMP2. (a) The procedure of harvesting and purification of CRMP2. (b) CRMP2 band of CBB staining after SDS-PAGE. (c) Result of WB.

\section{Results and discussion}

\subsection{Fundamental characteristics}

Figures 4(a) and 4(b) show the laser characteristics of the fabricated GaInAsP nanolaser in three solutions with different $\mathrm{pH}$ values. A single and sharp emission spectrum was observed above the laser threshold. The emission intensity showed a strong dependency on $\mathrm{pH}$. A low threshold was obtained particularly when the device was immersed in a low-pH solution. This is caused by the reduction of the negative charge via the acid dissociation equilibrium on the semiconductor surface, decreasing Schottky barrier near the semiconductor surface and decreasing surface recombination. The detailed mechanism is reported in our previous work (Sakemoto et al., 2016; Watanabe et al., 2015). The pH dependencies at a constant pump power are shown in Fig. 4(c). A high sensitivity was observed when the irradiated pump power $P_{\text {irr }}$ was set slightly above the 
threshold. This result can be explained by the process where electrons and holes excited by photopumping were separated in space by the Schottky barrier and that so-induced electric field compensated the barrier itself particularly at high pumping. Therefore, in the sensing experiment, it is desirable to set the pump power as low as possible under the condition that sufficient emission can be detected.

(a)

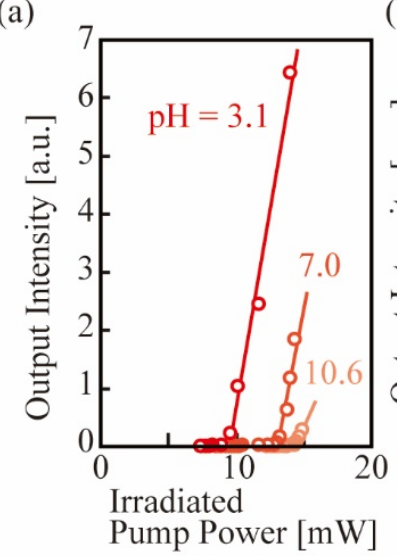

(b)

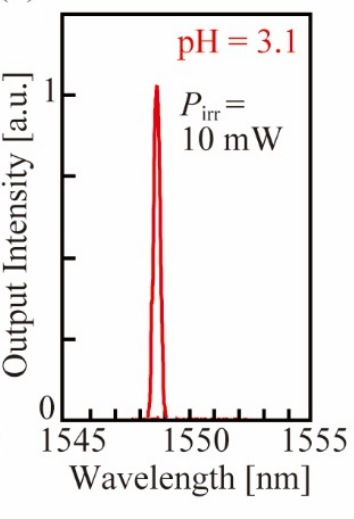

(c)

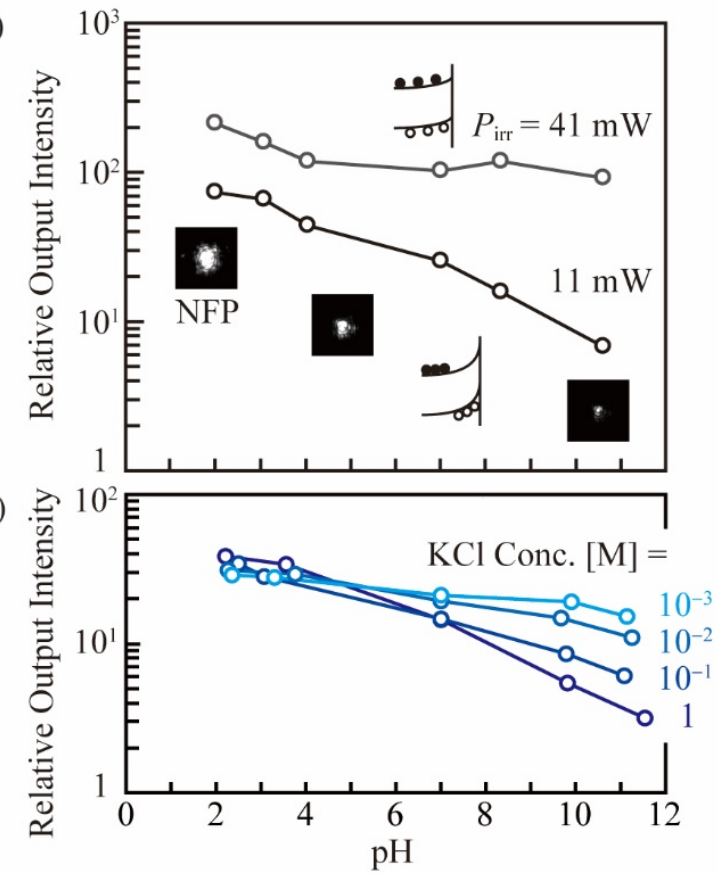

Fig. 4. Fundamental characteristics of the nanolaser biosensor. (a) Emission characteristics in solutions with different $\mathrm{pH}$ values. (b) Laser spectrum. (c) $\mathrm{pH}$ dependency of the laser emission intensity. Insets depict the schematic band lineup and distribution of photopumped carriers in a semiconductor at different pump powers. Near-field patterns (NFP) are also shown. (d) $\mathrm{pH}$ dependency for different $\mathrm{KCl}$ concentrations.

Compared with biosensors based on the PL intensity of semiconductors, our device is unique in respect of utilizing the laser emission intensity as a sensor signal. When the surface charge is changed, the surface recombination modifies the internal quantum efficiency $\eta_{\text {in. }}$. The PL intensity is simply changed by the ratio $r$ before and after the modification; that is,

$$
r=\eta_{\text {in }}{ }^{\prime} \eta_{\text {in }}
$$

In lasers, on the other hand, the ratio includes the threshold power $P_{\text {th }}$ as shown below:

$$
r=\eta_{\text {in }}{ }^{\prime}\left(P_{\text {irr }}-P_{\text {th }}{ }^{\prime}\right) / \eta_{\text {in }}\left(P_{\text {irr }}-P_{\text {th }}\right)=\left(\eta_{\text {in }}{ }^{\prime} P_{\text {irr }}-P_{\text {th } 0}\right) /\left(\eta_{\text {in }} P_{\text {irr }}-P_{\text {th } 0}\right)
$$

where $P_{\text {th } 0}$ is a constant value given by $P_{\text {th } 0}=\eta_{\text {in }}{ }^{\prime} P_{\text {th }}{ }^{\prime}$, showing the effective threshold. Therefore, when $P_{\text {irr }}$ is set appropriately so that the denominator or numerator of this equation takes a value close to zero, $r$ is arbitrarily 
increased or decreased, which is a significant advantage of lasers. In addition, GaInAsP semiconductors with a few nanometer oxide film have a good chemical stability compared with, for example, GaAs, for which the surface state is very unstable and its surface recombination is incomparably large in a solution having strong acid, base, and specific salt elements under photopumping (Nazemi et al., 2015; Wallys et al., 2012).

\subsection{Dependency of surface charge density}

Figure 4(d) shows the $\mathrm{pH}$ dependency of the laser emission for different $\mathrm{KCl}$ concentrations. In the $\mathrm{KCl}$ solution, the surface charge density is determined by the equilibrium constants of four states, i.e., $\mathrm{Zr}-\mathrm{O}^{-}$and $\mathrm{Zr}$ $\mathrm{OH}_{2}{ }^{+}$due to the ionization of surface hydroxyl groups that depend on $\mathrm{pH}$ as well as $\mathrm{Zr}-\mathrm{O}^{-} \mathrm{K}^{+}$and $\mathrm{Zr}-\mathrm{OH}_{2}{ }^{+} \mathrm{Cl}^{-}$ due to $\mathrm{K}^{+}$and/or $\mathrm{Cl}^{-}$complexation between ionic species and the $\mathrm{ZrO}_{2}$ surface (Davis et al., 1978). When the complexation cannot be neglected, the slope of $\mathrm{pH}$ dependency is changed particularly at a high $\mathrm{KCl}$ concentration. As seen from this figure, the emission intensity clearly depended on the $\mathrm{KCl}$ concentration. A high concentration yielded a large dependency. We consider that this behavior can be explained by the presence of the formation of surface complexation between $\mathrm{K}^{+}$and $\mathrm{Cl}^{-}$ions and $\mathrm{ZrO}_{2}$ surface (Janusz, 2000, 1988; Persin et al., 1992). One can also deduce the point of zero charge from the condition exhibiting no dependency on the salt concentration. The point of zero charge meaning a $\mathrm{pH}$ at which the surface charge is zero was estimated to be 4-5 in our device. This result is consistent with the point of zero charge of 4.3 at a $\mathrm{ZrO}_{2}$ surface in a $\mathrm{NaCl}$ solution estimated from a report (Janusz, 1988) using potentiometric titration. This correspondence supports the notion that the emission intensity of this nanolaser has the sensitivity to the surface charge density.

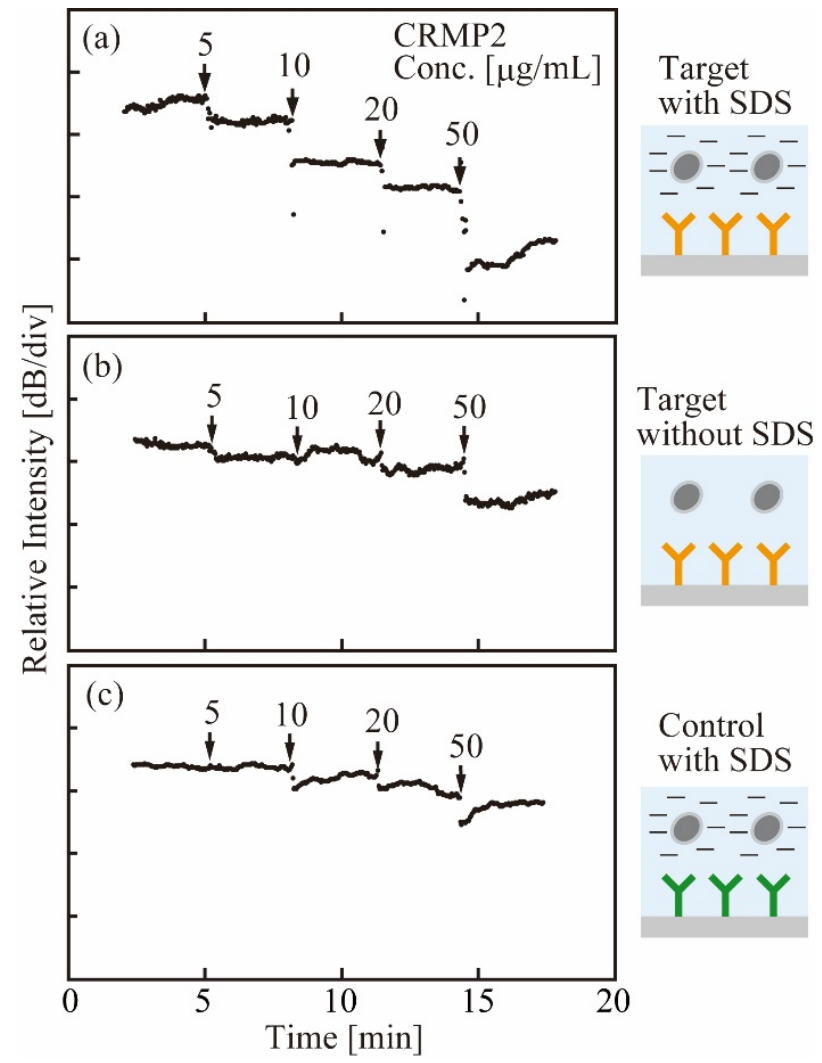

Fig. 5. Real-time measurement of laser emission intensity under three different conditions. (a) CRMP2 with SDS treatment. (b) CRMP2 without SDS treatment. (c) CRMP2 with SDS treatment, while the device was modified with the negative control antibody.

\subsection{Detection of CRMP2}

We first investigated the optimum SDS concentration for the treatment to CRMP2. As the SDS concentration applicable to the kit for removing excess SDS is from $0.1 \%$ to $1 \%$, we set the SDS concentration in this range. The emission due to the adsorption of several $\mu \mathrm{g} / \mathrm{mL}$ CRMP2 became larger as the SDS concentration gets higher. Therefore, we decided to set the SDS concentration to $1 \%$. We confirmed in the western-blot that the 
activity of CRMP2 was maintained even for this concentration. Figure 5 shows the real-time observation of the emission intensity. The behaviors of anti-CRMP2 and control antibodies with and without SDS were compared when the standard CRMP2 protein was used as the target. The emission intensity was obviously reduced after injecting a $5 \mu \mathrm{g} / \mathrm{mL}$ CRMP2 solution with SDS. Clear stepwise reduction was also observed at high concentrations. These results indicate that the surface was negatively charged and the emission intensity was reduced by the adsorption of CRMP2. The signals without SDS treatment or for the control antibody became obviously weak because of the small amount of negative charge or the absence of specific binding, respectively. It was also observed that signal reduction occurred rapidly after injecting the CRMP2 solution. The nanolaser might detect not only the adsorbed CRMP2 but also the protein inside the Debye length in the solution.

We repeated these experiments four to six times and obtained the average emission intensities under three different conditions, as shown in Fig. 6(a). Clear differences among the signals with SDS treatment and others were observed, particularly when the CRMP2 concentration was 5 and $10 \mu \mathrm{g} / \mathrm{mL}$. At high concentrations, the original charge effect of CRMP2 might be dominant even without the SDS treatment, and nonspecific signals were enhanced even for the control. Here, we also investigated the dependence of the emission intensity change on the SDS concentration. The intensity change obviously became larger for higher SDS concentrations. However, the plateau region at high CRMP2 concentrations was lying at above $50 \mu \mathrm{g} / \mathrm{ml}$, regardless of the SDS concentration. This means that the specific binding and nonspecific adsorption of CRMP2 are not accelerated by SDS even though the signal intensity is enhanced. We fit a 4-parameter logistic curve to each set of experimental plots, assuming that the adsorption of CRMP2 yields a general sigmoidal kinetic curve, as shown in Fig. 6(b). This result showed a correlation coefficient of $R^{2}=0.997$, which is sufficient as a standard curve for the quantitative evaluation. Even using linear fitting, the correlation coefficient of $R^{2}=0.97$ was confirmed, which may allow rough evaluation. We also checked the fluctuation in the sensitivity (for simplicity, we evaluated the $\mathrm{pH}$ sensitivity) between six different devices on a same chip and repeated it for four different chips, i.e., 24 devices in total. The sensitivity in $\mathrm{dB} / \mathrm{pH}$ and its fluctuation were obtained from the change in emission intensity of 24 nanolasers for three different $\mathrm{pH}$ solutions. The total average sensitivity was $0.60 \mathrm{~dB} / \mathrm{pH}$ and the standard deviation $\sigma$ was $0.092 \mathrm{~dB} / \mathrm{pH}$, for which $95 \%$ coverage of fluctuations was $\pm 1.96 \sigma= \pm 30 \%$ of the average value. It is seen from Fig. 6 that this fluctuation is sufficiently small to identify between the target and control.

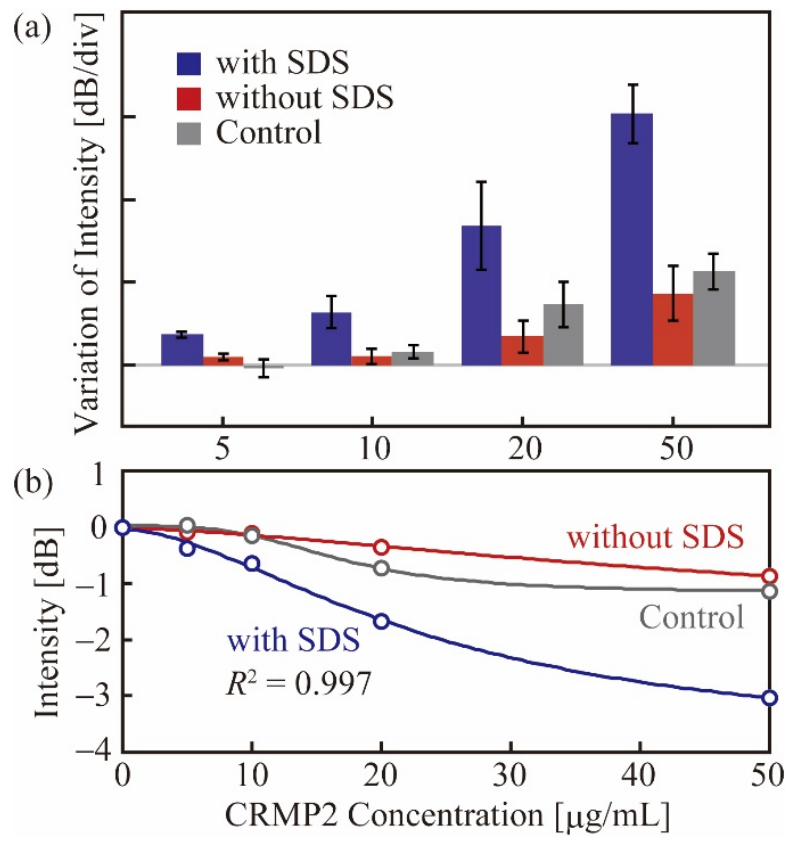

Fig. 6. Average variation of laser emission intensity for multiple experiments plotted as a function of CRMP2 concentration. Four experiments were carried out with and without SDS, and six experiments were carried for the control. (a) Comparison between three different conditions. The error bars represent standard errors in the average of each measurement. (b) The standard curve fitting of the experimental plots. 
In general, the limit of detection (LOD) is given by a concentration at which the signal becomes to be $3.3 \sigma$ (Shrivastava and Gupta, 2011). Here, we use $\sigma$ as the sum of the above value and the temporal fluctuation of the emission intensity in the real-time measurement, which was evaluated to be $0.19 \mathrm{~dB}$ for the measured intensity level in Fig. 5. Then, the LOD was evaluated to be $3.8 \mu \mathrm{g} / \mathrm{mL}$, which is comparable to the concentration of CRMP2 expressed in the peripheral lymphocytes of a healthy person. Although this LOD is not better than those of conventional western-blot, ELISA, and other label-free methods based on optical micro/nano-cavities, which require wavelength or angular spectral analyses (Chocarro-Ruiz et al., 2017; Gavela et al., 2016; Xavier et al., 2018), it satisfies the minimum requirement to detect the increase of CRMP2 from the diseases. The simple and quick detection using the nanolaser still maintains a great advantage. This LOD was evaluated for $1 \%$ SDS, while it was increased to 14 and $20 \mu \mathrm{g} / \mathrm{mL}$ for $0.5 \%$ and $0.2 \%$ SDS, respectively. This is the evidence that the sensitivity of this method is improved by adding SDS.

\section{Conclusion}

The emission intensity of ion-sensitive GaInAsP nanolaser biosensors was changed by the surface charge under the condition of a constant pump power. The biosensing signal can be detected by neither labels nor spectrum analyses but merely by measuring the emission intensity. Compared with similar studies exploiting the ion sensitivity of semiconductor photoluminescence, the response to surface charge can be enhanced by utilizing laser emission. This ion sensitivity is explained by the surface recombination modified by the change of Schottky barrier height. The sensing signal was increased when the pump power was moderately suppressed and set close to the laser threshold. Based on this principle, we demonstrated the detection of CRMP2 as a candidate biomarker for neuropsychiatric diseases, such as Alzheimer's disease and schizophrenia. The LOD was $3.8 \mu \mathrm{g} / \mathrm{mL}$, which corresponds to the concentration expressed in the peripheral blood of a healthy person. Therefore, the sensitivity is sufficient for medical diagnoses of these diseases with a much simpler procedure and much shorter duration, as compared with those of conventional western-blot and ELISA.

But still, the improvement of the sensitivity may be an issue for the practical use of this method. Although the nanolaser satisfies the minimum requirement, higher sensitivity will make this sensing more robust and the quantification more accurate even with additional fluctuations. Since the measurement is done at wavelengths longer than $1.3 \mu \mathrm{m}$, optical components are relatively expensive as compared with those at wavelengths shorter than $1.0 \mu \mathrm{m}$. Higher sensitivity also improves the $\mathrm{S} / \mathrm{N}$ and allows to use cheaper components. The development of low-cost compact micro-fluidic channel and a compact module of laser diode and photodiode will also become important.

\section{Acknowledgments}

We would like to thank Motokazu Koga, Aoi Jitsuki-Takahashi, Hiroko Makihara, and Takako Okada and Takeshi Kawashima for their expertise and technical support. This study was supported by Grant-in-Aid \#16H06334 from the Ministry of Education Culture, Sports, Science and Technology.

\section{References}

Budz, H.A., Ali, M.M., Li, Y., Lapierre, R.R., 2010. Photoluminescence model for a hybrid aptamer-GaAs optical biosensor. J. Appl. Phys. 107. https://doi.org/10.1063/1.3419714

Chocarro-Ruiz, B., Fernández-Gavela, A., Herranz, S., Lechuga, L.M., 2017. Nanophotonic label-free biosensors for environmental monitoring. Curr. Opin. Biotechnol. 45, 175-183. https://doi.org/10.1016/j.copbio.2017.03.016

Chow, E., Grot, A., Mirkarimi, L.W., Sigalas, M., Girolami, G., 2004. Ultracompact biochemical sensor built with two-dimensional photonic crystal microcavity. Opt. Lett. 29, 1093. https://doi.org/10.1364/OL.29.001093

Cole, A.R., Noble, W., Aalten, L. van, Plattner, F., Meimaridou, R., Hogan, D., Taylor, M., LaFrancois, J., Gunn-Moore, F., Verkhratsky, A., Oddo, S., LaFerla, F., Giese, K.P., Dineley, K.T., Duff, K., Richardson, J.C., Yan, S. Du, Hanger, D.P., Allan, S.M., Sutherland, C., 2007. Collapsin response mediator protein-2 hyperphosphorylation is an early event in Alzheimer's disease progression. J. Neurochem. 103, 1132-1144. https://doi.org/10.1111/j.1471-4159.2007.04829.x

Davis, J., James, R., Leckie, J., 1978. Surface Ionization and Complexation at the Oxide / Water Interface. J. 
Colloid Interface Sci. 63, 480-499. https://doi.org/10.1016/S0021-9797(78)80009-5

Ellis, A.B., Brainard, R.J., Kepler, K.D., Moore, D.E., Winder, E.J., Kuech, T.F., Lisensky, G.C., 1997. Modulation of the Photoluminescence of Semiconductors by Surface Adduct Formation : An Application of Inorganic Photochemistry to Chemical Sensing 74, 680-684. https://doi.org/10.1021/ed074p680

Gavela, A.F., García, D.G., Ramirez, J.C., Lechuga, L.M., 2016. Last advances in silicon-based optical biosensors. Sensors (Switzerland) 16, 1-15. https://doi.org/10.3390/s16030285

Goshima, Y., Nakamura, F., Strittmatter, P., Strittmatter, S.M., 1995. Collapsin-induced growth cone collapse mediated by an intracellular protein related to UNC-33. Nature 376, 509-514. https://doi.org/10.1038/376509a0

Hachuda, S., Watanabe, T., Takahashi, D., Baba, T., 2016. Sensitive and selective detection of prostatespecific antigen using a photonic crystal nanolaser. Opt. Express 24, 12886. https://doi.org/10.1364/OE.24.012886

Hideshima, S., Fujita, K., Harada, Y., Tsuna, M., Seto, Y., Sekiguchi, S., Kuroiwa, S., Nakanishi, T., Osaka, T., 2016. Signal amplification in electrochemical detection of buckwheat allergenic protein using field effect transistor biosensor by introduction of anionic surfactant. Sens. Bio-Sensing Res. 7, 90-94. https://doi.org/10.1016/j.sbsr.2016.01.011

Hong, Y., Huh, Y.M., Yoon, D.S., Yang, J., 2012. Nanobiosensors based on localized surface plasmon resonance for biomarker detection. J. Nanomater. 2012. https://doi.org/10.1155/2012/759830

Isono, T., Yamashita, N., Obara, M., Araki, T., Nakamura, F., Kamiya, Y., Alkam, T., Nitta, A., Nabeshima, T., Mikoshiba, K., Ohshima, T., Goshima, Y., 2013. Amyloid- $\beta 25-35$ induces impairment of cognitive function and long-term potentiation through phosphorylation of collapsin response mediator protein 2 . Neurosci. Res. 77, 180-185. https://doi.org/10.1016/j.neures.2013.08.005

Janusz, W., 2000. The Electrical Double Layer Parameters for the Group 4 Metal Oxide/Electrolyte System. Adsorpt. Sci. Technol. 18, 117-134. https://doi.org/10.1260/0263617001493332

Janusz, W., 1988. Surface charge and adsorption of $\mathrm{Na}+$ and $\mathrm{Cl}-$ ions at the zirconium dioxide/water interface. J. Radioanal. Nucl. Chem. Artic. 125, 393-401. https://doi.org/10.1007/BF02041697

Kita, S., Hachuda, S., Otsuka, S., Endo, T., Imai, Y., Nishijima, Y., Misawa, H., Baba, T., 2011a. Supersensitivity in label-free protein sensing using a nanoslot nanolaser. Opt. Express 19, 17683. https://doi.org/10.1364/OE.19.017683

Kita, S., Nozaki, K., Hachuda, S., Watanabe, H., Saito, Y., Otsuka, S., Nakada, T., Arita, Y., Baba, T., $2011 b$. Photonic crystal point-shift nanolasers with and without nanoslots - Design, fabrication, lasing, and sensing characteristics. IEEE J. Sel. Top. Quantum Electron. 17, 1632-1647. https://doi.org/10.1109/JSTQE.2011.2134837

Koczula, K.M., Gallotta, A., 2016. Lateral flow assays. Essays Biochem. 60, 111-120. https://doi.org/10.1042/EBC20150012

Kurrat, R., Prenosil, J.E., Ramsden, J.J., 1997. Kinetics of Human and Bovine Serum Albumin Adsorption at Silica-Titania Surfaces. J. Colloid Interface Sci. 185, 1-8. https://doi.org/10.1006/jcis.1996.4528

Makowski, M.S., Ivanisevic, A., 2011. Molecular analysis of blood with micro-/nanoscale field-effecttransistor biosensors. Small 7, 1863-1875. https://doi.org/10.1002/smll.201100211

Moulton, S.E., Barisci, J.N., McQuillan, A.J., Wallace, G.G., 2003. ATR-IR spectroscopic studies of the influence of phosphate buffer on adsorption of immunoglobulin G to TiO2. Colloids Surfaces A Physicochem. Eng. Asp. 220, 159-167. https://doi.org/10.1016/S0927-7757(03)00078-5

Narimatsu, M., Kita, S., Abe, H., Baba, T., 2012. Enhancement of vertical emission in photonic crystal nanolasers. Appl. Phys. Lett. 100. https://doi.org/10.1063/1.3696056

Nazemi, E., Aithal, S., Hassen, W.M., Frost, E.H., Dubowski, J.J., 2015. GaAs/AlGaAs heterostructure based photonic biosensor for rapid detection of Escherichia coli in phosphate buffered saline solution. Sensors Actuators, B Chem. 207, 556-562. https://doi.org/10.1016/j.snb.2014.10.111

Nomoto, M., 2018. Altered phosphorylation levels of collapsin response mediator protein 2 in peripheral blood samples from schizophrenia patients. Psychiatry Res.

Persin, M., Randon, J., Sarrazin, J., Larbot, A., Guizard, C., Cot, L., 1992. Influence of membrane-solution interface on the performance of zirconia ultrafiltration membrane. J. Colloid Interface Sci. 154, 416-422. https://doi.org/10.1016/0021-9797(92)90157-H

Sajid, M., Kawde, A.N., Daud, M., 2015. Designs, formats and applications of lateral flow assay: A literature review. J. Saudi Chem. Soc. 19, 689-705. https://doi.org/10.1016/j.jscs.2014.09.001

Sakemoto, M., Kishi, Y., Watanabe, K., Abe, H., Ota, S., Takemura, Y., Baba, T., 2016. Cell imaging using 
GaInAsP semiconductor photoluminescence. Opt. Express 24, 11232.

https://doi.org/10.1364/OE.24.011232

Shrivastava, A., Gupta, V., 2011. Methods for the determination of limit of detection and limit of quantitation of the analytical methods. Chronicles Young Sci. 2, 21. https://doi.org/10.4103/2229-5186.79345

Spielbauer, D., Mekhemer, G.A.H., Riemer, T., Zaki, M.I., Knözinger, H., 1997. Structure and Acidic Properties of Phosphate-Modified Zirconia. J. Phys. Chem. B 101, 4681-4688. https://doi.org/10.1021/jp963785x

Tandon, R., Gaebel, W., Barch, D.M., Bustillo, J., Gur, R.E., Heckers, S., Malaspina, D., Owen, M.J., Schultz, S., Tsuang, M., Van Os, J., Carpenter, W., 2013. Definition and description of schizophrenia in the DSM-5. Schizophr. Res. 150, 3-10. https://doi.org/10.1016/j.schres.2013.05.028

Tang, L., Chun, I.S., Wang, Z., Li, J., Li, X., Lu, Y., 2013. DNA detection using plasmonic enhanced nearinfrared photoluminescence of gallium arsenide. Anal. Chem. 85, 9522-9527. https://doi.org/10.1021/ac401169c

Tessier-Lavigne, M., Goodman, C.S., 1996. The molecular biology of axon guidance. Science 274, 1123-33.

Viter, R., Savchuk, M., Iatsunskyi, I., Pietralik, Z., Starodub, N., Shpyrka, N., Ramanaviciene, A., Ramanavicius, A., 2018. Analytical, thermodynamical and kinetic characteristics of photoluminescence immunosensor for the determination of Ochratoxin A. Biosens. Bioelectron. 99, 237-243. https://doi.org/10.1016/j.bios.2017.07.056

Vollmer, F., Braun, D., Libchaber, A., Khoshsima, M., Teraoka, I., Arnold, S., 2002. Protein detection by optical shift of a resonant microcavity. Appl. Phys. Lett. 80, 4057-4059. https://doi.org/10.1063/1.1482797

Wallys, J., Teubert, J., Furtmayr, F., Hofmann, D.M., Eickhoff, M., 2012. Bias-enhanced optical ph response of group III-nitride nanowires. Nano Lett. 12, 6180-6186. https://doi.org/10.1021/n1303021v

Watanabe, K., Baba, T., Nomoto, M., Nakamura, F., Goshima, Y., 2017. Label- and Spectral-Analysis-Free Detection of Neuropsychiatric Disease Biomarker Using, in: IEEE Sensors. Glasgow, UK, pp. 1-3. https://doi.org/10.1109/ICSENS.2017.8234350

Watanabe, K., Kishi, Y., Hachuda, S., Watanabe, T., Sakemoto, M., Nishijima, Y., Baba, T., 2015. Simultaneous detection of refractive index and surface charges in nanolaser biosensors. Appl. Phys. Lett. 106, 21106. https://doi.org/10.1063/1.4904481

Watanabe, T., Saijo, Y., Hasegawa, Y., Watanabe, K., Nishijima, Y., Baba, T., 2017. Ion-sensitive photoniccrystal nanolaser sensors. Opt. Express 25, 24469. https://doi.org/10.1364/OE.25.024469

Wei, T., Kaewtathip, S., Shing, K., 2009. Buffer Effect on Protein Adsorption at Liquid/Solid Interface. J. Phys. Chem. C 113, 2053-2062. https://doi.org/10.1021/jp806586n

Weickert, C.S., Weickert, T.W., Pillai, A., Buckley, P.F., 2013. Biomarkers in schizophrenia: A brief conceptual consideration. Dis. Markers 35, 3-9. https://doi.org/10.1155/2013/510402

Xavier, J., Vincent, S., Meder, F., Vollmer, F., 2018. Advances in optoplasmonic sensors - Combining optical nano/microcavities and photonic crystals with plasmonic nanostructures and nanoparticles. Nanophotonics 7, 1-38. https://doi.org/10.1515/nanoph-2017-0064

Yamashita, N., Ohshima, T., Nakamura, F., Kolattukudy, P., Honnorat, J., Mikoshiba, K., Goshima, Y., 2012. Phosphorylation of CRMP2 (Collapsin Response Mediator Protein 2) Is Involved in Proper Dendritic Field Organization. J. Neurosci. 32, 1360-1365. https://doi.org/10.1523/JNEUROSCI.5563-11.2012

Yoshinobu, T., Iwasaki, H., Ui, Y., Furuichi, K., Ermolenko, Y., Mourzina, Y., Wagner, T., Näther, N., Schöning, M.J., 2005. The light-addressable potentiometric sensor for multi-ion sensing and imaging. Methods 37, 94-102. https://doi.org/10.1016/j.ymeth.2005.05.020 